\begin{tabular}{|c|l|}
\hline Title & Indecisive behavior of amoeba crossing an environmental barrier \\
\hline Author(s) & Takagi, S.; Nishiura, Y.; Nakagaki, T.; U eda, T.; U eda, K.-I. \\
\hline Citation & Topological A spects of Critical Systems and Networks: Proceedings of the International Symposium, 86-93 \\
\hline Issue Date & 2007-06 \\
\hline Doc URL & http://hdl.handle.net/2115/40062 \\
\hline Type & proceedings (author version) \\
\hline Note & $\begin{array}{l}\text { International Symposium on "Topological A spects of Critical Systems and Networks". 13-14 February 2006. Hokkaido } \\
\text { University, Sapporo, JA PAN. }\end{array}$ \\
\hline File Information & nishiura15.pdf \\
\hline
\end{tabular}

Instructions for use 


\title{
Indecisive behavior of amoeba crossing an environmental barrier *
}

\author{
S. Takagi, Y. Nishiura, T. Nakagaki, T. Ueda \\ Research Institute for Electronic Science, Hokkaido University, \\ Sapporo 060-0812, Japan \\ E-mail: takagi@es.hokudai.ac.jp

\section{K.-I.Ueda} \\ Research Institute for Mathematical Sciences, Kyoto University, \\ Sakyo-ku, Kyoto, 606-8502, Japan \\ E-mail: ueda@kurims.kyoto-u.ac.jp
}

\begin{abstract}
We report here a new kind of behavior that seems to be 'indecisive' in an amoeboid organism, the Physarum plasmodium of true slime mold. The plasmodium migrating in a narrow lane stops moving for a period of time (several hours but the duration differs for each plasmodium) when it encounters the presence of a chemical repellent, quinine. After stopping period, the organism suddenly begins to move again in one of three different ways as the concentration of repellent increases: going through the repulsive place (penetration), splitting into two fronts of going throught it and turning (splitting) and turning back (rebound). In relation to the physiological mechanism for tip migration in the plasmodium, we found that the frontal tip is capable of moving further although the tip is divided from a main body of organism. This means that a motive force of front locomotion is produced by a local process at the tip. Based on this finding, a mathematical model for front locomotion is considered in order to understand the dynamics for both the long period of stopping and three kinds of behavior. A model based on reaction-diffusion equations succeeds to reproduce the experimental observation. The origin of long-time stopping and three different outputs may be reduced to the hidden instabilities of internal dynamics of the pulse, which may be a skeleton structure extracted from much more complex dynamics imbedded in the Physarum plasmodium.
\end{abstract}

*This work is supported by Grant-in-aid for Scientific Researchof the Japan Society for the Promotion of Science. 


\section{Introduction}

So far behaviors of unicellular organism was expected to be simple and trivial. This is because there is no brain and nervous system in unicellular organism. In a higher animal, behaviors are determined as an output from information processing in a brain. However, an amoeba shows rich behaviors. In fact, a biologist, H. S. Jennings, already reported a hundred years ago that it is possible for us to feel the similar sort of feeling to a Paramecium as that to a dog if it would be as large as a dog. ${ }^{1}$

The plasmodium of Physarum polycephalum, true slime mold, is a large aggregate of protoplasm and crawls by amoeboid movement. This organism shows smart behaviors and its dynamical aspect is partially studied: tactic movement to stimulation ${ }^{2,3}$ and maze solving. ${ }^{4-7}$ Information processing is performed by the protoplasm in the plasmodium because of no brain. So cell dynamics is closely related to how smart behaviors emerge. In this sense, the plasmodium is a useful model organism to study information processing at cell level. Here we report a new kind of amoeboid behavior that seems to be indecisive behavior in the plasmodium. Here we define indecisive behavior as: when an organism encounters a different situation or a problem, it is unable to make a clear decision on a course of action for a period of time, and only being to act once a decision is made. A mathematical model for the behavior is proposed and discussion is made on how indecisive-like behavior appears in terms of nonlinear dynamics.

In the next section, we show the experiments and the results on the behaviors of plasmodium and on the locomotion activity of the frontal tip. Then we describe a mathematical model of pulse dynamics described in one-dimensional reaction diffusion system. The numerical results give that pulse dynamics shows almost the same behaviors as those of plasmodium.

\section{Experiment}

\subsection{Materials and Methods}

The plasmodium of Physarum polycephalum (strain HU195 $\times$ HU200) was fed with oatmeal on a $1 \%$ agar gel at $25{ }^{\circ} \mathrm{C}$ in the dark. Fixed amount, $100 \mathrm{mg}$, of the extending front of the cultured plasmodium was cut off and placed at an edge of an agar gel $(1.5 \%)$ lane of $1 \mathrm{~cm}$ in width and 30 $\mathrm{cm}$ in length. The prepared plasmodia exhibited almost one-dimensional locomotion and protoplasm distribution (see Figure 1(a)). For the repellent region, an agar gel block containing quinine was embedded at the middle of a lane. The length of the repellent region was fixed at $10 \mathrm{~mm}$ and the 
a)

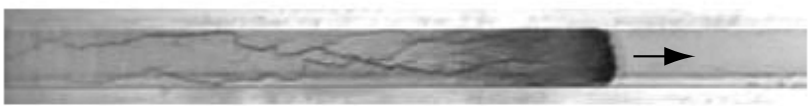

b)

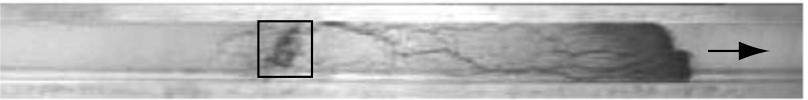

c)

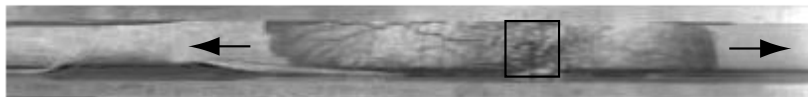

d)

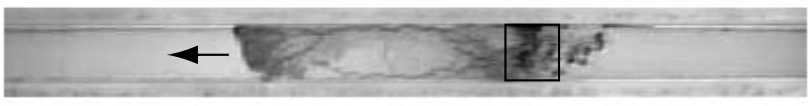

Fig. 1. Repellent in the middle of a lane causes a variety of cell behavior as its concentration varies. The arrows indicate the locomotion directions, and rectangles represent the regions containing a repellent. In each case, the plasmodia migrated from the left side in the figure. (a) A plasmodium migrating in a one-dimensional lane without repellent (control). (b) Penetration ([quinine $]=4 \mathrm{mM}$ ), (c) splitting ([quinine $]=6 \mathrm{mM}$ ), (d) rebound ([quinine] $=8 \mathrm{mM}$ ). The width of the lane is $1 \mathrm{~cm}$ and the length of the repellent region is $1 \mathrm{~cm}$.

concentration of the repellent (quinine) was varied as 4,6 and $8 \mathrm{mM}$. The experiment was done at $25{ }^{\circ} \mathrm{C}$ in dark condition. The movement of the plasmodia was monitored by the following method. The plasmodia were illuminated from below with an infrared ray $(\lambda \sim 950 \mathrm{~nm})$, the wavelength of which is harmless for the plasmodium, ${ }^{8}$ and the transmitted light image was observed by time laps video camera, and analyzed on a personal computer. The place of the frontal tip of a migrating plasmodium was detected by subtracting the background image.

\subsection{Experimental result}

We found that the plasmodia exhibits 3 types of cell behavior depending on the concentration of quinine. The snapshots of the plasmodia exhibiting each response and the time traces of the places of the frontal tips are shown in Figures 1 and 2, respectively. In each case, the plasmodium stopped at the repellent region for several hours after coming up there, i.e., they were indecisive for a period of time, and finally exhibited various behaviors. When the quinine concentration was low $(4 \mathrm{mM})$, the plasmodium went through the repellent region after about 6 hours indecision (Figure 2(a)). When the 

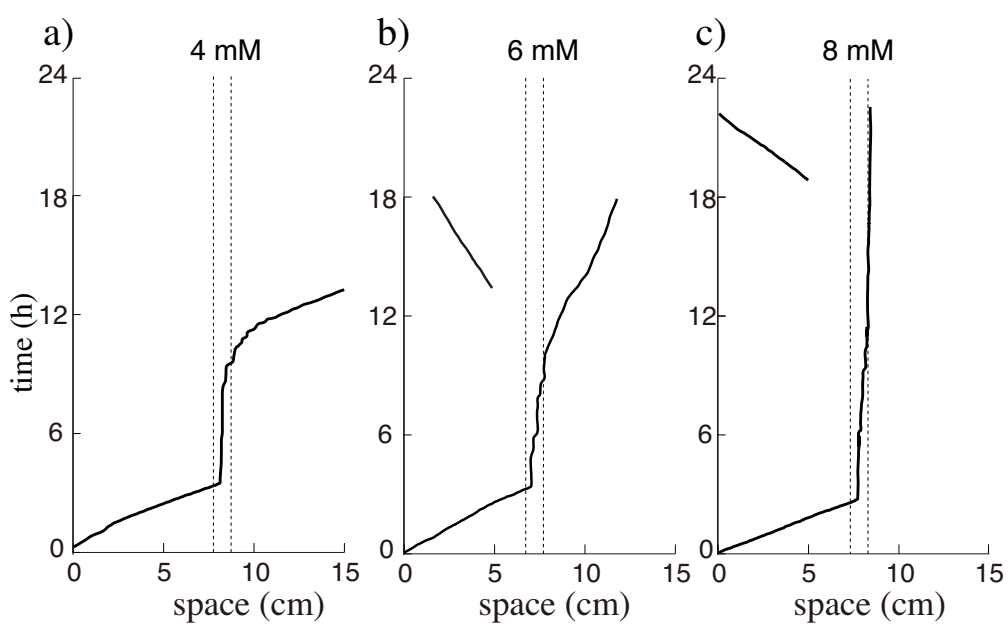

Fig. 2. Tip trajectories for each reaction (a) penetration, (b) splitting and (c) rebound. Experimental conditions are the same as Figure 1.

quinine concentration was high $(8 \mathrm{mM})$, the plasmodium turned back from the repellent region after about 15 hours indecision (Figure 2(c)). At the intermediate quinine concentration $(6 \mathrm{mM})$, the plasmodium exhibited both responses, that is, one part of the plasmodium went through the repellent region and the other part returned back; thus the locomotion front split into two fronts (Figure 2(b)). When the plasmodium returns back, since the frontal tip is created around the end of the tail of the plasmodium, the returning tip trajectory starts at a point apart from the repellent region. In the case of splitting, decrease in the locomotion velocity after splitting is due to halving of the protoplasm, since locomotion velocity decreases as the total mass of the protoplasm decreases. ${ }^{9}$ Here we note the following facts: around the condition in which the plasmodium shows splitting behavior, the behavior is not uniquely determined, that is, other behavior, penetration or rebound, is also observed under the same conditions; duration of indecision differs for each plasmodium even under the same condition.

\subsection{Experiment on the frontal tip activity of locomotion}

Here we describe only the essentials of the method and the result of the experiment to examine the locomotion activity of the frontal tip. A plasmodium was allowed to migrate in a narrow lane, similarly to the experiment above, and the frontal tip was divided from main body by cutting with a 
surgical knife, that is, amputation of the frontal tip. The place of the amputation from the tip, $d$, was varied from 0.8 to $5 \mathrm{~mm}$ in the plasmodium with the length of $\sim 90 \mathrm{~mm}$, and the mean extension speed of the tip was measured before the amputation, $v_{0}$, and after the amputation, $v_{a}$.

Figure 3(a) shows the space-time plot of the thickness profile of a plasmodium. After the amputation, the tip extends at the almost the same velocity as before the amputation, while the rhythmic contraction disappears. The extension velocity is roughly constant from 5 to $1 \mathrm{~mm}$ amputation (Figure 3(b)). It should be noted that the decrease of the extension velocity at small $d$ can be caused by the damage due to the amputation. This result suggests that the rhythmic contraction is not a motive force of locomotion and extension of the frontal tip, and the frontal locomotion will be driven by a process localized at the tip.
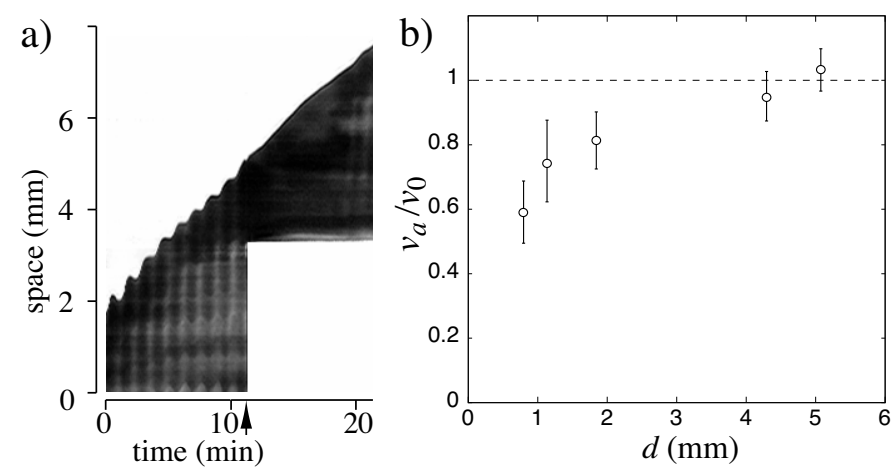

Fig. 3. Amputation of the tip to examine the locomotion activity of the frontal tip. (a) Space-time plot of thickness profile of a plasmodium directionally migrating in a narrow lane. The thickness is represented by grayscale; darker color corresponds to thicker protoplasm. The plasmodium migrates from bottom to top. At the timing indicated by the arrow, the frontal tip was divided from the main body that was removed after the amputation. Origins of the axes are determined arbitrarily. (b) Ratio of the locomotion velocities before, $v_{0}$, and after, $v_{a}$, the amputation, $v_{a} / v_{0}$.

\section{Mathematical model}

We obtained clear evidence that the locomotion process is localized at the frontal tip. In other word, a tip having the locomotion activity is able to be the locomotion front. Besides, it was reported that polarized intracellular chemical pattern corresponds to the polarity of a plasmodium, e.g., ATP 
concentration is higher at the locomotion front and becomes lower toward the tail. ${ }^{10,11}$ According to these facts, we consider the dynamics of polarized chemical pulse based on one-dimensional reaction diffusion system called Gray-Scott model, ${ }^{12}$ in which we assume that the chemical pattern with pulse-like form corresponds to the activity of the locomotion front.

$$
\begin{aligned}
& \frac{\partial u}{\partial t}=D_{u} \frac{\partial^{2} u}{\partial x^{2}}-u v^{2}+f(1-u), \\
& \frac{\partial v}{\partial t}=D_{v} \frac{\partial^{2} v}{\partial x^{2}}+u v^{2}-(f+k(x)) v,
\end{aligned}
$$

where $u=u(t, x)$ and $v=v(t, x)$ depend on time $t$ and position $x, f>0$ and $k>0$ are kinetic parameters, and diffusion coefficients $D_{u}>0, D_{v}>0$ are constants. In this model, $u$ and $v$ represent substrate and product concentrations, respectively. Therefore, in relation to the Physarum plasmodium, we can consider that $u$ and $v$ correspond to nutrients and intracellular ATP concentrations, respectively. $k(x)$ is a perturbed parameter with heterogeneous distribution of bump type:

$$
k(x)=\left\{\begin{array}{l}
k_{0}, \quad x \in\left[0, x_{0}\right) \cup\left(x_{1}, L\right], \\
k_{0}+\epsilon, \quad x \in\left[x_{0}, x_{1}\right],
\end{array}\right.
$$

where $k_{0}, \epsilon \in \mathbb{R}$. Note that $\epsilon$ denotes the height of the bump. For the case of single jump-discontinuity, a detailed analysis has been done by Dynamics of traveling pulses in heterogeneous media of jump type. ${ }^{13}$ We integrate Equations 1 using the Crank-Nicholson method with $D_{u} / D_{v}=2, L=$ $4.0, x_{1}-x_{0}=0.2$ and time interval $\Delta t=0.05$ with Neumann boundary conditions. In this study we fix the parameter $f=0.024$. Figure 4 shows that the pulse exhibits three different responses when the pulse encounters a bump in the middle after stopping on it for a period of time, namely, the pulse shows the indecisive behavior. For low bump, the pulse can penetrate the bump after stopping period. For high bump, the pulse cannot penetrate the bump and turns back after stopping period. For intermediate bump, the pulse splits into two pulses, and one of them penetrates and the other returns back. Therefore, the pulse exhibits qualitatively the same behaviors as the plasmodium. The phase diagram of the pulse dynamics is shown in Figure 5. The pulse shows penetration when $\epsilon$ is small, and it shows rebound when $\epsilon$ is large. When $k_{0}$ is small, there is a splitting region between the penetration and the rebound regions. Consequently, there are three behaviors for the pulse dynamics in the heterogeneous media of bump type, and the pulse changes its behavior depending on the parameter values of $k_{0}$ and $\epsilon$. Here we can give a correspondence between model parameters 
$\left(k_{0}\right.$ and $\left.\epsilon\right)$ and experimental conditions: $k_{0}$ represents the environmental conditions, i.e, temperature, humidity, agar concentration and so on, and $\epsilon$ represents the repellent concentration at the middle of a lane, e.g., quinine concentration; higher $k_{0}$ and $\epsilon$ values correspond to worse condition. We have obtained good correspondence between $\epsilon$ and quinine concentration for the pulse and the plasmodium behavior.
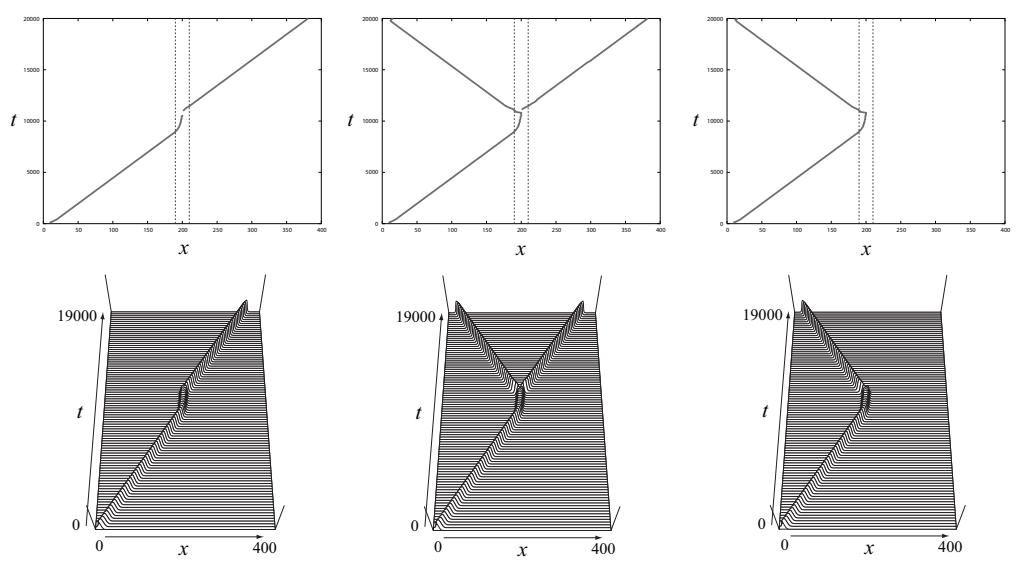

Fig. 4. Pulse dynamics of Gray-Scott model (Equations 1) in heterogeneous media with different bump heights. Upper panel shows time traces of the wave fronts, and lower panel shows space-time plots of pulse dynamics. Left: Penetration $(\epsilon=0.000216614)$. Middle: splitting $(\epsilon=0.000216618)$. Right: rebound $(\epsilon=0.000216619)$. In each case $k_{0}=0.05543$. The bump $\left(\left[x_{0}, x_{1}\right], x_{0}=1.9, x_{1}=2.1\right)$ is indicated by dotted lines.

\section{Discussion}

Even in an amoeba, indecisive-like behaviors are observed. Although the indecisive behavior is thought to be a higher function in the brain, it is possible at cell level. Capacity of information processing in a cell is much higher than expected. So further evaluation is needed.

Here we discuss a possible mechanism of indecisive-like behavior in terms of nonlinear dynamics. A key idea in the mathematical model proposed here is characterized by a recently suggested new concept of scattor (see, for instance, Nishiura et al., Chaos $(2003)^{14}$ ): a saddle-like structure which attracts a wide-open trajectories and sorts them out to several basins of different attractors. Loosely speaking, scattor corresponds to indecisive state and each different attractor corresponds to a different pulse behavior. As 


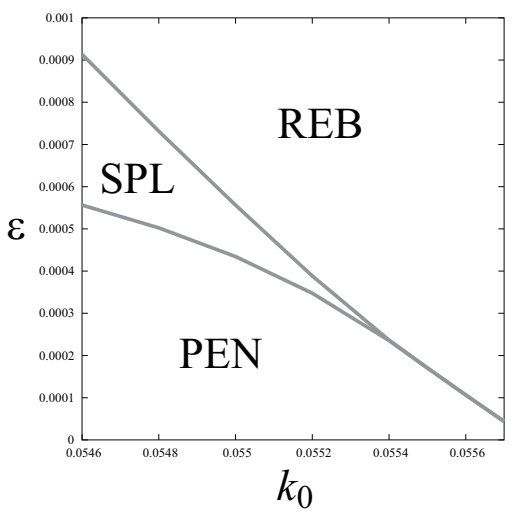

Fig. 5. Phase diagram of pulse dynamics for heterogeneous media. PEN: penetration, SPL: splitting, REB: rebound. Triple junction is $\left(k_{0}, \epsilon\right) \approx(0.0555,0.0001705)$. The place of the bump is the same as in Figure 4

parameters vary, closer to the scattor, longer the time for a trajectory to stay as the indecisive state. But the duration of time in real experiments is rather unpredictable under a small fluctuation, nevertheless the scattor dynamics as above could be regarded as a minimal dynamical model which can reproduce the observed behaviors in the real plasmodium. In this sense, this model may grasp a mathematical essence for the development of indecisive-like behaviors in true slime mold at higher abstraction level.

\section{References}

1. H. S. Jennings Amer. J. Psycol. 10, No.4 (1899).

2. K. Matsumoto, T. Ueda and Y. Kobatake J. Theor. Biol. 122, 339 (1986).

3. K. Matsumoto, T. Ueda and Y. Kobatake J. Theor. Biol. 131, 175 (1988).

4. T. Nakagaki, H. Yamada and Á. Tóth, Nature 407, 470 (2000).

5. T. Nakagaki, H. Yamada and Á. Tóth, Biophys. Chem. 92, 47 (2001).

6. A. Tero, R. Kobayashi, T. Nakagaki, Physica A363, 115 (2006).

7. A. Tero, R. Kobayashi, T. Nakagaki, J. Theor. Biol. accepted.

8. T. Ueda, Y. Mori, T. Nakagaki, Y. Kobatake, Photochem. Photobiol. 48, 705 (1988).

9. T. Ueda in Networks of Interacting Machines, chap. 9, eds. D. Armbruster, K. Kaneko, A. S. Mikhailov (World Scientific, 2005).

10. Y. Mori, K. Matsumoto, T. Ueda, Y. Kobatake, Protoplasma 135, 31 (1986).

11. T. Ueda, Y. Mori, Y. Kobatake, Exp. Cell Res. 169, 191 (1987).

12. P. Gray, S. K. Scott, J. Phys. Chem 89, 22 (1985).

13. Y. Nishiura, Y. Oyama, K.-I. Ueda, to appear in Hokkaido Math.J.

14. Y. Nishiura, T. Teramoto, K. -I. Ueda, Chaos 13, 962 (2003) 